\title{
Structure-Preserving Model Order Reduction of RCL Circuit Equations
}

\author{
Roland W. Freund \\ Department of Mathematics, University of California at Davis, One Shields \\ Avenue, Davis, CA 95616, U.S.A. \\ freund@math.ucdavis.edu
}

Summary. In recent years, order-reduction techniques based on Krylov subspaces have become the methods of choice for generating macromodels of large multi-port RLC circuits. Despite the success of these techniques and the extensive research efforts in this area, for general RCL circuits, the existing Krylov subspace-based reduction algorithms do not fully preserve all essential structures of the given large RCL circuit. In this paper, we describe the problem of structure-preserving model order reduction of general RCL circuits, and we discuss two state-of-the-art algorithms, PRIMA and SPRIM, for the solution of this problem. Numerical results are reported that illustrate the higher accuracy of SPRIM vs. PRIMA. We also mention some open problems.

\section{Introduction}

Electronic circuits often contain large linear subnetworks of passive components. Such subnetworks may represent interconnect (IC) automatically extracted from layout as large RCL networks, models of IC packages, or models of wireless propagation channels. Often these subnetworks are so large that they need to be replaced by much smaller reduced-order models, before any numerical simulation becomes feasible. Ideally, these models would produce a good approximation of the input-output behavior of the original subnetwork, at least in a limited domain of interest, e.g., a frequency range.

In recent years, reduced-order modeling techniques based on Padé or Padétype approximation have been recognized to be powerful tools for various circuit simulation tasks. The first such technique was asymptotic waveform evaluation (AWE) [31], which uses explicit moment matching. More recently, the attention has moved to reduced-order models generated by means of Krylovsubspace algorithms, which avoid the typical numerical instabilities of explicit moment matching; see, e.g., the survey papers [14, 15, 16].

PVL $[9,10]$ and its multi-port version MPVL [11] use variants of the Lanczos process [26] to stably compute reduced-order models that represent Padé 
or matrix-Padé approximations [5] of the circuit transfer function. SyPVL [21] and its multi-port version SyMPVL [12, 23, 24] are versions of PVL and MPVL, respectively, that are tailored to RCL circuits. By exploiting the symmetry of RCL transfer functions, the computational costs of SyPVL and SyMPVL are only half of those of general PVL and MPVL.

Reduced-order modeling techniques based on the Arnoldi process [3], which is another popular Krylov-subspace algorithm, were first proposed in $[33,28,8,29,30]$. Arnoldi-based reduced-order models are defined by a certain Padé-type approximation property, rather than Padé approximation, and as a result, in general, they are not as accurate as a Padé-based model of the same size. In fact, Arnoldi-based models are known to match only half as many moments as Lanczos-based models; see [33, 28, 29, 15].

In many applications, in particular those related to VLSI interconnect, the reduced-order model is used as a substitute for the full-blown original model in higher-level simulations. In such applications, it is very important for the reduced-order model to maintain the passivity properties of the original circuit. In $[23,24,4]$, it is shown that SyMPVL is passive for RC, RL, and LC circuits. However, the Padé-based reduced-order model that characterizes SyMPVL cannot be guaranteed to be passive for general RCL circuits. On the other hand, in [28, 29,30], it was proved that the Arnoldi-based reduction technique PRIMA produces passive reduced-order for general RCL circuits. PRIMA employs a block version of the Arnoldi process and then obtains reduced-order models by projecting the matrices defining the RCL transfer function onto the Arnoldi basis vectors. While PRIMA generates provably passive reduced-order models, it does not preserve other structures, such as reciprocity or the block structure of the circuit matrices, inherent to RCL circuits. This has motivated the development of the reduction technique SPRIM [17, 18], which overcomes these disadvantages of PRIMA. In particular, SPRIM generates provably passive and reciprocal macromodels of multi-port RCL circuits. Furthermore, SPRIM models match twice as many moments as the corresponding PRIMA models obtained with identical computational work. In this paper, we describe the problem of structure-preserving model order reduction of general RCL circuits, and we discuss the PRIMA and SPRIM algorithms for the solution of this problem.

The remainder of this article is organized as follows. In Section 2, we review the formulation of general RCL circuits as systems of integro-differentialalgebraic equations (integro-DAEs). In Section 3, we describe the problem of structure-preserving model order reduction of systems of integro-DAEs. In Section 4, we present an equivalent formulation of such systems as timeinvariant linear dynamical systems. In Section 5, we review oder reduction based on projection onto Krylov subspaces and the PRIMA algorithm. In Section 6, we describe the SPRIM algorithm for order reduction of general RCL circuits, and in Section 7, we present some theoretical properties of SPRIM. In Section 8 , we report the results of some numerical experiments with SPRIM 
and PRIMA. Finally, in Section 9, we mention some open problems and make some concluding remarks.

Throughout this article the following notation is used. The set of real and complex numbers is denoted by $\mathbb{R}$ and $\mathbb{C}$, respectively. Unless stated otherwise, all vectors and matrices are allowed to have real or complex entries. For (real or complex) matrices $M=\left[m_{j k}\right]$, we denote by $M^{T}=\left[m_{k j}\right]$ the transpose of $M$, and by $M^{*}:=\left[\overline{m_{k j}}\right]$ the Hermitian (or complex conjugate) of $M$. The identity matrix is denoted by $I$ and the zero matrix by 0 ; the actual dimensions of $I$ and 0 will always be apparent from the context. The notation $M \succeq 0(M \succ 0)$ is used to indicate that a real or complex square matrix $M$ is Hermitian positive semidefinite (positive definite). If all entries of the matrix $M \succeq 0(M \succ 0)$ are real, then $M$ is said to be symmetric positive semidefinite (positive definite). The kernel (or null space) of a matrix $M$ is denoted by ker $M$.

\section{Formulation of general RCL circuits as integro-DAEs}

In this section, we review the formulation of general RCL circuits as systems of integro-DAEs.

\subsection{Electronic circuits as directed graphs}

We use the standard lumped-element approach that models general electronic circuits as directed graphs; see, e.g., [7, 34]. More precisely, a given circuit is described as a directed graph $\mathcal{G}=(\mathcal{N}, \mathcal{E})$ whose edges $e \in \mathcal{E}$ correspond to the circuit elements and whose nodes $n \in \mathcal{N}$ correspond to the interconnections of the circuit elements. For each element for which the direction of the current flow through the element is known beforehand, the corresponding edge is oriented in the direction of the current flow; for example, current sources and voltage sources are elements with known direction of current flow. For all other elements, arbitrary directions are assigned to the edges corresponding to these elements. Each edge $e \in \mathcal{E}$ can be written as an ordered pair of nodes, $e=\left(n_{1}, n_{2}\right)$, where the direction of $e$ is from node $n_{1}$ to node $n_{2}$. We say that the edge $e=\left(n_{1}, n_{2}\right)$ leaves node $n_{1}$ and enters node $n_{2}$.

The directed graph $\mathcal{G}=(\mathcal{N}, \mathcal{E})$ can be described by its incidence matrix $\mathcal{A}=\left[a_{j k}\right]$. The rows and columns of $\mathcal{A}$ correspond to the nodes and edges of the directed graph, respectively, and the entries $a_{j k}$ of $\mathcal{A}$ are defined as follows:

$$
a_{j k}= \begin{cases}1 & \text { if edge } e_{k} \text { leaves node } n_{j} \\ -1 & \text { if edge } e_{k} \text { enters node } n_{j}, \\ 0 & \text { otherwise }\end{cases}
$$

In order to avoid redundancy, any one of the nodes can be selected as the datum (or ground) node of the circuit. We denote by $n_{0}$ the datum node, 
by $\mathcal{N}_{0}=\mathcal{N} \backslash\left\{n_{0}\right\}$ the remaining non-datum nodes, and by $\mathcal{A}_{0}$ the matrix obtained by deleting from $\mathcal{A}$ the row corresponding to $n_{0}$. Note that $\mathcal{A}_{0}$ is called the reduced incidence matrix of the directed graph $\mathcal{G}$. We remark that $\mathcal{A}_{0}$ has full row rank, i.e.,

$$
\operatorname{rank} \mathcal{A}_{0}=\left|\mathcal{N}_{0}\right|
$$

provided the graph $\mathcal{G}$ is connected; see, e.g., [7, Theorem 9-6].

We denote by $v=v(t)$ the vector of nodal voltages at the non-datum nodes $\mathcal{N}_{0}$, i.e., the $k$-th entry of $v$ is the voltage at node $n_{k} \in \mathcal{N}_{0}$. We denote by $v_{\mathcal{E}}=v_{\mathcal{E}}(t)$ and $i_{\mathcal{E}}=i_{\mathcal{E}}(t)$ the vectors of edge voltages and currents, respectively, i.e., the $j$-th entry of $v_{\mathcal{E}}$ is the voltage across the circuit element corresponding to edge $e_{j} \in \mathcal{E}$, and the $j$-th entry of $i_{\mathcal{E}}$ is the current through the circuit element corresponding to edge $e_{j} \in \mathcal{E}$.

Any general electronic circuit is described completely by three types of equations: Kirchhoff's current laws (KCLs), Kirchhoff's voltage laws (KVLs), and the branch constitutive relations (BCRs); see, e.g., [34]. The KCLs state that at each node $n \in \mathcal{N}$, the currents along all edges leaving and entering the node $n$ sum up to zero. In terms of the reduced incidence matrix $\mathcal{A}_{0}$ of $\mathcal{G}$ and the vector $i_{\mathcal{E}}$, the KCLs can be expressed in the following compact form:

$$
\mathcal{A}_{0} i_{\mathcal{E}}=0 .
$$

Similarly, the KCVs state that for any closed (undirected) loop in the graph $\mathcal{G}$, the voltages along the edges of the loop sum up to zero. The KCLs can be expressed in the following compact form:

$$
\mathcal{A}_{0}^{T} v=v_{\mathcal{E}} .
$$

The BCRs are the equations that describe the physical behavior of the circuit elements.

\subsection{RCL circuit equations}

We now restrict ourselves to general linear RCL circuits. The possible element types of such circuits are resistors, capacitors, inductors, independent voltage sources, and independent current sources. We use subscripts $r, c, l, v$, and $i$ to denote edge quantities corresponding to resistors, capacitors, inductors, voltage sources, and current sources of the RCL circuit, respectively. Moreover, we assume that the edges $\mathcal{E}$ are ordered such that we have the following partitionings of the reduced incidence matrix and the vectors of edge voltages and currents:

$$
\mathcal{A}_{0}=\left[\begin{array}{lllll}
\mathcal{A}_{r} & \mathcal{A}_{c} & \mathcal{A}_{l} & \mathcal{A}_{v} & \mathcal{A}_{i}
\end{array}\right], \quad v_{\mathcal{E}}=\left[\begin{array}{c}
v_{r} \\
v_{c} \\
v_{l} \\
v_{v} \\
v_{i}
\end{array}\right], \quad i_{\mathcal{E}}=\left[\begin{array}{c}
i_{r} \\
i_{c} \\
i_{l} \\
i_{v} \\
i_{i}
\end{array}\right] .
$$


The BCRs for the resistors, capacitors, and inductors can be expressed in the following compact form:

$$
v_{r}(t)=R i_{r}(t), \quad i_{c}(t)=C \frac{d}{d t} v_{c}(t), \quad v_{l}(t)=L \frac{d}{d t} i_{l}(t) .
$$

Here,

$$
R \succ 0, \quad C \succ 0, \quad \text { and } \quad L \succ 0
$$

are symmetric positive definite matrices. Furthermore, $R$ and $C$ are diagonal matrices whose diagonal entries are the resistances and capacitances of the resistors and capacitors, respectively. The diagonal entries of $L$ are the inductances of the inductors. Often $L$ is also diagonal, but in general, when mutual inductances are included, $L$ is not diagonal. The BCRs for the voltage sources simply state that $v_{v}(t)$ is a given input vector, the entries of which are the voltages provided by the voltages sources. Similarly, the BCRs for the current sources state that $i_{i}(t)$ is a given input vector, the entries of which are the currents provided by the current sources.

The KCLs (1), the KVLs (2), and the BCRs (4), together with initial conditions for the nodal voltages $v\left(t_{0}\right)$ at some initial time $t_{0}$, describe the behavior of a given RCL circuit. Without loss of generality, we set $t_{0}=0$. The initial condition then reads

$$
v(0)=v^{(0)},
$$

where $v^{(0)}$ is a given vector. Moreover, for simplicity, we also assume that

$$
i_{l}(0)=0 \text {. }
$$

Then, the BCRs for the inductors in (4) can be equivalently stated as follows:

$$
i_{l}(t)=L^{-1} \int_{0}^{t} v_{l}(\tau) d \tau
$$

The resulting set of equations describing a given RCL circuit can be simplified considerably by eliminating the edge quantities corresponding to the resistors, capacitors, and inductors. To this end, we first use the partitionings (3) to rewrite the KCLs (1) as follows:

$$
\mathcal{A}_{r} i_{r}+\mathcal{A}_{c} i_{c}+\mathcal{A}_{l} i_{l}+\mathcal{A}_{v} i_{v}+\mathcal{A}_{i} i_{i}=0 .
$$

Similarly, the KCVs (2) can be expressed as follows:

$$
\mathcal{A}_{r}^{T} v=v_{r}, \quad \mathcal{A}_{c}^{T} v=v_{c}, \quad \mathcal{A}_{l}^{T} v=v_{l}, \quad \mathcal{A}_{v}^{T} v=v_{v}, \quad \mathcal{A}_{i}^{T} v=v_{i} .
$$

From (4), (7), and (9), it follows that

$$
\begin{aligned}
& i_{r}(t)=R^{-1} \mathcal{A}_{r}^{T} v(t), \quad i_{c}(t)=C \mathcal{A}_{c}^{T} \frac{d}{d t} v(t), \\
& i_{l}(t)=L^{-1} \mathcal{A}_{l}^{T} \int_{0}^{t} v(\tau) d \tau .
\end{aligned}
$$


Inserting (10) into (8), and using (9), we obtain

$$
\begin{aligned}
M_{11} \frac{d}{d t} v(t)+D_{11} v(t)+\mathcal{A}_{v} i_{v}(t)+K_{11} \int_{0}^{t} v(\tau) d \tau & =-\mathcal{A}_{i} i_{i}(t), \\
-\mathcal{A}_{v}^{T} v(t) & =-v_{v}(t), \\
v_{i}(t) & =\mathcal{A}_{i}^{T} v(t),
\end{aligned}
$$

where

$$
M_{11}:=\mathcal{A}_{c} C \mathcal{A}_{c}^{T}, \quad D_{11}:=\mathcal{A}_{r} R^{-1} \mathcal{A}_{r}^{T}, \quad K_{11}:=\mathcal{A}_{l} L^{-1} \mathcal{A}_{l}^{T} .
$$

The equations (11) can be viewed as a linear dynamical system for the unknown state-space vector

$$
z(t):=\left[\begin{array}{c}
v(t) \\
i_{v}(t)
\end{array}\right]
$$

with given input vector and unknown output vector

$$
u(t):=\left[\begin{array}{c}
-i_{i}(t) \\
v_{v}(t)
\end{array}\right] \quad \text { and } \quad y(t):=\left[\begin{array}{c}
v_{i}(t) \\
-i_{v}(t)
\end{array}\right]
$$

respectively. Indeed, setting

$$
\begin{array}{ll}
M:=\left[\begin{array}{cc}
M_{11} & 0 \\
0 & 0
\end{array}\right], \quad D:=\left[\begin{array}{cc}
D_{11} & \mathcal{A}_{v} \\
-\mathcal{A}_{v}^{T} & 0
\end{array}\right], \\
K:=\left[\begin{array}{cc}
K_{11} & 0 \\
0 & 0
\end{array}\right], \quad F:=\left[\begin{array}{cc}
\mathcal{A}_{i} & 0 \\
0 & -I
\end{array}\right], \quad z^{(0)}:=\left[\begin{array}{c}
v^{(0)} \\
i_{v}(0)
\end{array}\right],
\end{array}
$$

and using (13), (14), and (9), the equations (11) can be rewritten in the form

$$
\begin{aligned}
M \frac{d}{d t} z(t)+D z(t)+K \int_{0}^{t} z(\tau) d \tau & =F u(t), \\
y(t) & =F^{T} z(t),
\end{aligned}
$$

and the initial conditions (6) can be stated in the form

$$
z(0)=z^{(0)} \text {. }
$$

Note that, in (16), $M, D$, and $K$ are $N_{0} \times N_{0}$ matrices and $F$ is an $N_{0} \times m$ matrix. Here, $N_{0}$ is the sum of the number of non-datum nodes in the circuit and the number of voltage sources, and $m$ denotes the number of all voltage and current sources. We remark that $N_{0}$ is the state-space dimension of the linear dynamical system (16), and $m$ is the number of inputs (and outputs) of (16). In general, the matrix $M$ is singular, and thus the first equation of (16) is a system of integro-differential-algebraic equations (integro-DAEs). Finally, note that the matrices (15), $M, D, K$, and $F$, exhibit certain structures. In particular, from (5), (12), and (15), it follows that

$$
M=\left[\begin{array}{cc}
M_{11} & 0 \\
0 & 0
\end{array}\right] \succeq 0, \quad D+D^{T}=\left[\begin{array}{cc}
2 D_{11} & 0 \\
0 & 0
\end{array}\right] \succeq 0, \quad \text { and } \quad K \succeq 0 .
$$




\section{Structure-preserving model order reduction}

In this section, we formulate the problems of model order reduction and structure preservation.

\subsection{Model order reduction}

A reduced-order model of the linear dynamical system (16) is a system of the same form as (16), but with smaller state-space dimension $n_{0}\left(<N_{0}\right)$. More precisely, a reduced-order model of (16) with state-space dimension $n_{0}$ is a system of the form

$$
\begin{aligned}
\tilde{M} \frac{d}{d t} \tilde{z}(t)+\tilde{D} \tilde{z}(t)+\tilde{K} \int_{0}^{t} \tilde{z}(\tau) d \tau & =\tilde{F} u(t), \\
\tilde{y}(t) & =\tilde{F}^{T} \tilde{z}(t),
\end{aligned}
$$

with initial conditions

$$
\tilde{z}(0)=\tilde{z}^{(0)}
$$

where

$$
\tilde{M}, \tilde{D}, \tilde{K} \in \mathbb{R}^{n_{0} \times n_{0}}, \quad \tilde{F} \in \mathbb{R}^{n_{0} \times m}, \quad \text { and } \quad \tilde{z}^{(0)} \in \mathbb{R}^{n_{0}} .
$$

The general problem of order reduction of a given linear dynamical system (16) is to determine a reduced state-space dimension $n_{0}$ and data (19) such that the corresponding reduced-order model (18) is a sufficiently accurate approximation of (16).

A practical way of assessing the accuracy of reduced-order models is based on the concept of Laplace-domain transfer functions of linear dynamical systems. The transfer function of the original linear dynamical system (16) is given by

$$
H(s)=F^{T}\left(s M+D+\frac{1}{s} K\right)^{-1} F .
$$

Here, we assume that the matrix $s M+D+\frac{1}{s} K$ is singular only for finitely many values of $s \in \mathbb{C}$. Conditions that guarantee this assumption are given in Section 4 below.

In analogy to (20), the transfer function of a reduced-order model (18) of (16) is given by

$$
\tilde{H}(s)=\tilde{F}^{T}\left(s \tilde{M}+\tilde{D}+\frac{1}{s} \tilde{K}\right)^{-1} \tilde{F}
$$

Note that both

$$
H: \mathbb{C} \mapsto(\mathbb{C} \cup \infty)^{m \times m} \quad \text { and } \quad \tilde{H}: \mathbb{C} \mapsto(\mathbb{C} \cup \infty)^{m \times m}
$$

are $m \times m$-matrix-valued rational functions.

In terms of transfer functions, the problem of order reduction of the original system (16) is equivalent to the problem of constructing the matrices $\tilde{M}, \tilde{D}$, $\tilde{K}$, and $\tilde{F}$ in (18) such that the transfer function $(21), \tilde{H}(s)$, is a 'sufficiently accurate' approximation to the original transfer function $(20), H(s)$. 


\subsection{Structure preservation}

Recall that the linear dynamical system (16) with data matrices given in (15) describes the behavior of a given RCL circuit. Therefore, the reduced-order model (18) should be constructed such that it corresponds to an actual RCL circuit. This is the problem of structure-preserving model order reduction of RCL circuits: Generate matrices $\tilde{M}, \tilde{D}, \tilde{K}$, and $\tilde{F}$ such that the reduced-order model (18) can be synthesized as an RCL circuit. Obviously, (18) corresponds to an actual RCL circuit if the matrices $\tilde{M}, \tilde{D}, \tilde{K}$, and $\tilde{F}$ are constructed such that they have analogous structures as the matrices $M, D, K$, and $F$ of the original given RCL circuit. Unfortunately, for general RCL circuits, no order reduction method is known that is guaranteed to preserve all these 'RCL structures'. However, the SPRIM algorithm described in Section 6 below does generate reduced-order models (18) with matrices $\tilde{M}, \tilde{D}, \tilde{K}$, and $\tilde{F}$ that preserve the block structure (15) of the original matrices $M, D, K$, and $F$, as well as the semidefiniteness properties (17) of $M, D$, and $K$.

For the special cases of RC, RL, and LC circuits, there are variants of the general MPVL (Matrix-Padeé Via Lanczos) method [11] that do preserve the RC, RL, and LC structures, respectively. In particular, the SyPVL and SyMPVL algorithms are procedures for for generating reduced-order models that can be synthesized as RC, RL, and LC circuits, respectively; see [22, 23, $24]$.

\subsection{Passivity}

An important property of general RCL circuits is passivity. Roughly speaking, a system is passive if it does not generate energy. In particular, any RCL circuit is passive. For linear dynamical systems of the form (16), passivity is equivalent to positive realness of the associated transfer function (20), $H(s)$; see, e.g., $[2,30]$. The general definition of positive realness is as follows.

Definition 1. An $m \times m$-matrix-valued function $H: \mathbb{C} \mapsto(\mathbb{C} \cup \infty)^{m \times m}$ is called positive real if the following three conditions are satisfied:

(i) $H$ is analytic in $\mathbb{C}_{+}:=\{s \in \mathbb{C} \mid \operatorname{Re} s>0\}$;

(ii) $H(\bar{s})=\overline{H(s)}$ for all $s \in \mathbb{C}$;

(iii) $H(s)+(H(s))^{*} \succeq 0$ for all $s \in \mathbb{C}_{+}$.

Since any RCL circuit is passive, positive realness of the reduced-order transfer function $(21), \tilde{H}(s)$, is a necessary condition for the associated reduced-order model (18) to be synthesizable as an actual RCL circuit. However, in general, positive realness of $\tilde{H}(s)$ is not a sufficient condition. Nevertheless, any reduced-order model (18) with a positive real transfer function (21) can be synthesized as an actual physical electronic circuit, but it may contain other electronic devices besides resistors, capacitors, and inductors. We refer the reader to [2] for a discussion of the problem of synthesis of positive real transfer functions. 


\section{Equivalent first-order form of integro-DAEs}

The system of integro-DAEs (16) can also be formulated as an equivalent first-order system. In this section, we discuss such a first-order formulation and some of its properties.

\subsection{First-order formulation}

Consider equations (11) and their equivalent statement (16) as a system of integro-DAEs. It turns out that (11) (and thus (16)) can be rewritten as a first-order time-invariant linear dynamical system of the form

$$
\begin{aligned}
E \frac{d}{d t} x(t) & =A x(t)+B u(t), \\
y(t) & =B^{T} x(t),
\end{aligned}
$$

with initial conditions

$$
x(0)=x^{(0)} .
$$

Indeed, by adding the vector of inductance currents, $i_{l}(t)$, to the original statespace vector $(13), z(t)$, and using the last relation of $(10)$, one readily verifies that the equations (11) can be stated in the form (22) with data matrices, state-space vector, and initial vector given by

$$
\begin{aligned}
& A:=-\left[\begin{array}{ccc}
D_{11} & \mathcal{A}_{l} & \mathcal{A}_{v} \\
-\mathcal{A}_{l}^{T} & 0 & 0 \\
-\mathcal{A}_{v}^{T} & 0 & 0
\end{array}\right], \quad E:=\left[\begin{array}{ccc}
M_{11} & 0 & 0 \\
0 & L & 0 \\
0 & 0 & 0
\end{array}\right], \\
& B:=\left[\begin{array}{cc}
\mathcal{A}_{i} & 0 \\
0 & 0 \\
0 & -I
\end{array}\right], \quad x(t):=\left[\begin{array}{c}
v(t) \\
i_{l}(t) \\
i_{v}(t)
\end{array}\right], \text { and } x^{(0)}:=\left[\begin{array}{c}
v^{(0)} \\
0 \\
i_{v}(0)
\end{array}\right] .
\end{aligned}
$$

Here, $M_{11}$ and $D_{11}$ are the matrices defined in (12). Moreover, $A, E \in \mathbb{R}^{N \times N}$, $B \in \mathbb{R}^{N \times m}$, and $x^{(0)} \in \mathbb{R}^{N \times m}$, where $N$ denotes the state-space dimension of the system (22). We remark that $N$ is the sum of the state-space dimension $N_{0}$ of the equivalent system of integro-DAEs (16) and the number of inductors of the RCL circuit. Note that, in (22), the input vector $u(t)$ and the output vector $y(t)$ are the same as in (16), namely the vectors defined in (14). In particular, both systems (16) and (22) have $m$ inputs and $m$ outputs.

\subsection{Regularity of the first-order matrix pencil}

Next, we consider the matrix pencil

$$
s E-A, \quad s \in \mathbb{C}
$$


where $A$ and $E$ are the matrices defined in (23). The pencil (24) is said to be regular if the matrix $s E-A$ is singular only for finitely many values of $s \in \mathbb{C}$. In this subsection, we present conditions for regularity of (24).

In view of the definitions of $A$ and $E$ in (23), we have

$$
s E-A=\left[\begin{array}{ccc}
s M_{11}+D_{11} & \mathcal{A}_{l} & \mathcal{A}_{v} \\
-\mathcal{A}_{l}^{T} & s L & 0 \\
-\mathcal{A}_{v}^{T} & 0 & 0
\end{array}\right] \quad \text { for all } s \in \mathbb{C} .
$$

Now assume that $s \neq 0$, and set

$$
U_{1}(s)=\left[\begin{array}{ccc}
I & -\frac{1}{s} \mathcal{A}_{l} L^{-1} & 0 \\
0 & I & 0 \\
0 & 0 & I
\end{array}\right] \quad \text { and } \quad U_{2}(s)=\left[\begin{array}{ccc}
I & 0 & 0 \\
\frac{1}{s} L^{-1} \mathcal{A}_{l}^{T} & I & 0 \\
0 & 0 & I
\end{array}\right] .
$$

Then, one readily verifies that, for all $s \neq 0$,

$$
U_{1}(s)(s E-A) U_{2}(s)=\left[\begin{array}{ccc}
s M_{11}+D_{11}+\frac{1}{s} K_{11} & 0 & \mathcal{A}_{v} \\
0 & s L & 0 \\
-\mathcal{A}_{v}^{T} & 0 & 0
\end{array}\right],
$$

where $K_{11}$ is the matrix defined in (12).

We now use the relation (27) to establish a necessary and sufficient condition for regularity of (24). Recall from (3) that $\mathcal{A}_{r}, \mathcal{A}_{c}, \mathcal{A}_{l}$, and $\mathcal{A}_{v}$ are the submatrices of the reduced incidence matrix $\mathcal{A}_{0}$ corresponding to the resistors, capacitors, inductors, and voltage sources of the RCL circuit, respectively.

Theorem 1. (Regularity of the matrix pencil (24).)

(a) The pencil (24) is regular if, and only if, the matrix-valued function

$$
F(s):=\left[\begin{array}{cc}
F_{11}(s) & \mathcal{A}_{v} \\
-\mathcal{A}_{v}^{T} & 0
\end{array}\right], \text { where } F_{11}(s):=s M_{11}+D_{11}+\frac{1}{s} K_{11},
$$

is regular, i.e., the matrix $F(s)$ is singular only for finitely many values of $s \in \mathbb{C}, s \neq 0$.

(b) The pencil (24) is regular if, and only if, the matrix $\mathcal{A}_{v}$ has full column rank and the matrix

$$
\mathcal{A}_{1}:=\left[\begin{array}{llll}
\mathcal{A}_{r} & \mathcal{A}_{c} & \mathcal{A}_{l} & \mathcal{A}_{v}
\end{array}\right]
$$

has full row rank.

Proof. Part (a) readily follows from (27) and the fact that, in view of (5), the matrix $L \succ 0$ is nonsingular. Indeed, since the matrices (26), $U_{1}(s)$ and $U_{2}(s)$, are nonsingular for all $s \neq 0$, it follows from (27) that the pencil (24) is regular if, and only if, the matrix-valued function on the right-hand side 
of (27) is regular. Since $L$ is nonsingular, it follows that for the matrix-valued function (27) is regular if, and only if, $F(s)$, is regular.

To prove part (b), we make use of part (a). and we show that $F(s)$ is regular if, and only if $\mathcal{A}_{v}$ has full column rank and the matrix $\mathcal{A}_{1}$ has full row rank. Suppose $\mathcal{A}_{v}$ does not have full column rank, and let $c \neq 0$ a nontrivial vector in $\operatorname{ker} \mathcal{A}_{v}$. Then

$$
F(s)\left[\begin{array}{l}
0 \\
c
\end{array}\right]=0, \quad\left[\begin{array}{l}
0 \\
c
\end{array}\right] \neq 0,
$$

and thus $F(s)$ is singular for all $s$. Therefore, we can assume that

$$
\operatorname{ker} \mathcal{A}_{v}=\{0\} \text {. }
$$

Next, note that the function $s \operatorname{det} F(s)$ is a polynomial in $s$, and thus $F(s)$ is regular unless $\operatorname{det} F(s)=0$ for all $s$. Therefore, it is sufficient to consider the matrix (28), $F(s)$, for $s>0$ only. Using (12) and the definition (29) of $\mathcal{A}_{1}$, the submatrix $F_{11}(s)$ of $F(s)$ can be expressed as follows:

$$
F_{11}(s)=\left[\begin{array}{lll}
\mathcal{A}_{r} & \mathcal{A}_{c} & \mathcal{A}_{l}
\end{array}\right]\left[\begin{array}{ccc}
s C & 0 & 0 \\
0 & R^{-1} & 0 \\
0 & 0 & \frac{1}{s} L^{-1}
\end{array}\right]\left[\begin{array}{lll}
\mathcal{A}_{r} & \mathcal{A}_{c} & \mathcal{A}_{l}
\end{array}\right]^{T} .
$$

In view of (5), the $3 \times 3$ block diagonal matrix in (31) is symmetric positive definite for $s>0$. It follows that for all $s>0$, we have

$$
F_{11}(s) \succeq 0 \quad \text { and } \quad \operatorname{ker}\left(F_{11}(s)\right)=\operatorname{ker}\left(\left[\begin{array}{lll}
\mathcal{A}_{r} & \mathcal{A}_{c} & \mathcal{A}_{l}
\end{array}\right]^{T}\right) .
$$

Finally, we apply Theorem 3.2 from [6], which gives a necessary and sufficient condition for the nonsingularity of $2 \times 2$ block matrices of the form (28) with subblocks satisfying (30) and the first condition in (32). By [6, Theorem 3.2], it follows that for $s>0$, the matrix $F(s)$ is nonsingular if, and only if,

$$
\operatorname{ker}\left(F_{11}(s)\right) \cap \operatorname{ker}\left(\mathcal{A}_{v}^{T}\right)=\{0\} .
$$

Using the second relation in (32), we can rewrite (33) as follows:

$$
\left(\operatorname{ker}\left(\mathcal{A}_{1}^{T}\right)=\right) \operatorname{ker}\left(\left[\begin{array}{llll}
\mathcal{A}_{r} & \mathcal{A}_{c} & \mathcal{A}_{l} & \mathcal{A}_{v}
\end{array}\right]^{T}\right)=\{0\} .
$$

This condition is equivalent to the matrix (29), $\mathcal{A}_{1}$, having full row rank, and thus the proof of part (b) is complete.

Remark 1. In terms of the given RCL circuit, the rank conditions in part (b) of Theorem 1 have the following meaning. In view of (3), the matrix (29), $\mathcal{A}_{1}$, is the reduced incidence matrix of the subcircuit obtained from the given RCL circuit by deleting all independent current sources. This matrix has full row rank if this subcircuit is connected; see, e.g., [7, Theorem 9-6]. The matrix $\mathcal{A}_{v}$ is the reduced incidence matrix of the subcircuit consisting of only the independent voltage sources. This matrix has full column rank if this subcircuit does not contain any closed (undirected) loops; see, e.g., [7, Section 9-8]. 
Since the two circuit conditions in Remark 1 are satisfied for any practical RCL circuit, from now on, we assume that the matrix pencil (24) is regular.

\subsection{First-order transfer function}

In analogy to (20), the transfer function of the first-order formulation (22) is the matrix-valued rational function given by

$$
H: \mathbb{C} \mapsto(\mathbb{C} \cup \infty)^{m \times m}, \quad H(s)=B^{T}(s E-A)^{-1} B .
$$

We remark that (34) is a well-defined rational function since the matrix pencil $s E-A$ is assumed to be regular. Recall that the system of integro-DAEs (16) and its first-order formulation (22) have the same input and output vectors. Since transfer functions only depend on the input-output behavior of the system, it follows that the transfer functions (20) and (34) are identical, i.e.,

$$
\begin{aligned}
H(s) & =B^{T}(s E-A)^{-1} B \\
& =F^{T}\left(s M+D+\frac{1}{s} K\right)^{-1} F \quad \text { for all } \quad s \in \mathbb{C} .
\end{aligned}
$$

Here, $A, E, B$ and $M, D, K, F$ are the matrices given in (23) and (15), respectively. In particular, the regularity of the matrix pencil $s E-A$ also guarantees the existence of the transfer function (20) of the system of integroDAEs (16).

Remark 2. The relation (35) can also be verified directly using the identity (27), (26), the definition of the matrix $B$ in (23), and the definitions of the matrices $M, D, K$, and $F$ in (15).

Remark 3. The definitions of $A$ and $E$ in (23), together with (5) and (17), imply that

$$
-A-A^{*} \succeq 0 \text { and } \quad E \succeq 0 .
$$

The matrix properties (36) in turn guarantee that the transfer function (34), $H$ satisfies all conditions of Definition 1, and thus $H$ is positive real.

\subsection{Reduced-oder models}

A reduced-order model of the linear dynamical system (22) is a system of the same form as (22), but with smaller state-space dimension $n(<N)$. More precisely, a reduced-order model of (22) with state-space dimension $n$ is a system of the form

$$
\begin{aligned}
E_{n} \frac{d}{d t} \tilde{x}(t) & =A_{n} \tilde{x}(t)+B_{n} u(t), \\
\tilde{y}(t) & =B_{n}^{T} \tilde{x}(t),
\end{aligned}
$$


with initial conditions

$$
\tilde{x}(0)=\tilde{x}^{(0)}
$$

where $A_{n}$ and $E_{n}$ are $n \times n$ matrices, $B_{n}$ is an $n \times m$ matrix, and $\tilde{x}^{(0)}$ is a vector of length $n$.

Provided that the reduced-order matrix pencil

$$
s E_{n}-A_{n}, \quad s \in \mathbb{C},
$$

is regular, the transfer function of the reduced-order model (37) is given by

$$
H_{n}: \mathbb{C} \mapsto(\mathbb{C} \cup \infty)^{m \times m}, \quad H_{n}(s)=B_{n}^{T}\left(s E_{n}-A_{n}\right)^{-1} B_{n} .
$$

\section{Krylov-subspace projection and PRIMA}

In this section, we review the generation of reduced-order models (37) via projection, in particular onto block Krylov subspaces.

\subsection{Order reduction via projection}

A simple approach to model order reduction is to use projection. Let

$$
V_{n} \in \mathbb{C}^{N \times n}, \quad \operatorname{rank} V_{n}=n,
$$

be any given matrix with full column rank. Then, by setting

$$
A_{n}:=V_{n}^{*} A V_{n}, \quad E_{n}:=V_{n}^{*} E V_{n}, \quad B_{n}:=V_{n}^{*} B
$$

one obtains reduced data matrices that define a reduced-order model (37). From (36) and (42), it readily follow that

$$
-A_{n}-A_{n}^{*} \succeq 0 \text { and } \quad E_{n} \succeq 0 .
$$

If in addition, the matrix $V_{n}$ is chosen as a real matrix and the matrix pencil (39) is assumed to be regular, then the reduced-order transfer function (40), $H_{n}$, satisfies all conditions of Definition 1, and thus $H_{n}$ is positive real; see [15, Theorem 13].

\subsection{Block Krylov subspaces}

The simple projection approach (42) yields powerful model-order reduction techniques when the columns of the matrix (41), $V_{n}$, are chosen as basis vectors of certain block Krylov subspaces.

To this end, let $s_{0} \in \mathbb{C}$ be a suitably chosen expansion point such that the matrix $s_{0} E-A$ is nonsingular. Note that, in view of the regularity of the 
matrix pencil (24), there are only finitely many values of $s_{0}$ for which $s_{0} E-A$ is singular. We can then rewrite the transfer function (34), $H$, as follows:

$$
\begin{aligned}
H(s) & =B^{T}\left(s_{0} E-A+\left(s-s_{0}\right) E\right)^{-1} B \\
& =B^{T}\left(I+\left(s-s_{0}\right) M\right)^{-1} R,
\end{aligned}
$$

where

$$
M:=\left(s_{0} E-A\right)^{-1} E, \quad R:=\left(s_{0} E-A\right)^{-1} B .
$$

We will use block Krylov subspaces induced by the matrices $M$ and $R$ in (45) to generate reduced-order models.

Next, we briefly review the notion of block Krylov subspaces; see [1] for a more detailed discussion. The matrix sequence

$$
R, M R, M^{2} R, \ldots, M^{j-1} R, \ldots
$$

is called a block Krylov sequence. The columns of the matrices in this sequence are vectors of length $N$, and thus at most $N$ of these columns are linearly independent. By scanning the columns of the matrices in the block Krylov sequence from left to right and deleting each column that is linearly dependent on earlier columns, we obtain the deflated block Krylov sequence

$$
R_{1}, M R_{2}, M^{2} R_{3}, \ldots, M^{j-1} R, \ldots, M^{j_{\max }-1} R_{j_{\max }} .
$$

This process of deleting linearly dependent vectors is called deflation. In (46), each $R_{j}$ is a submatrix of $R_{j-1}$. Denoting by $m_{j}$ the number of columns of $R_{j}$, we thus have

$$
m \geq m_{1} \geq m_{2} \geq \cdots \geq m_{j} \geq \cdots \geq m_{j_{\max }} \geq 1 .
$$

By construction, the columns of the matrices (46) are linearly independent, and for each $n$, the subspace spanned by the first $n$ of these columns is called the $n$-th block Krylov subspace (induced by $M$ and $R$ ) and denoted by $\mathcal{K}_{n}(M, R)$ in the sequel.

For $j=1,2, \ldots, j_{\max }$, we set

$$
n(j):=m_{1}+m_{2}+\cdots+m_{j} .
$$

For $n=n(j)$, the $n$-th block Krylov subspace is given by

$$
\mathcal{K}_{n}(M, R)=\operatorname{colspan}\left[\begin{array}{lllll}
R_{1} & M R_{2} & M^{2} R_{3} & \cdots & M^{j} R_{j}
\end{array}\right] .
$$

Here and in the sequel, we use colspan $V$ to denote the subspace spanned by the columns of the matrix $V$. Finally, we remark that, by $(47), n(j) \leq m \cdot j$ with $n(j)=m \cdot j$ if no deflation has occurred. 


\subsection{Projection onto block Krylov subspaces and PRIMA}

PRIMA [28, 29, 30] combines projection with block Krylov subspaces. More precisely, the $n$-th PRIMA reduced-order model is defined by (37) and (42), where the matrix (41), $V_{n}$, is chosen such that its columns span the $n$-th block Krylov subspace $\mathcal{K}_{n}(M, R)$, i.e., colspan $V_{n}=\mathcal{K}_{n}(M, R)$. We refer to any such matrix $V_{n}$ as a basis matrix of the $n$-th Krylov subspace $\mathcal{K}_{n}(M, R)$.

Although the PRIMA reduced-order models are defined by simple projection, the combination with block Krylov subspaces guarantees that the PRIMA reduced-order models satisfy a Padé-type approximation property. For the special case $s_{0}=0$ and basis vectors generated by a block Arnoldi process without deflation, this Padé-type approximation property was first observed in [28]. In [13], this result was extended to the most general case where possibly nonzero expansion points $s_{0}$ are allowed and where the underlying block Krylov method allows the necessary deflation of linearly dependent vectors. The result can be stated as follows; for a proof, we refer the reader to $[15$, Theorem 7$]$.

Theorem 2. Let $n=n(j)$ be of the form (48) for some $1 \leq j \leq j_{\max }$, and let $V_{n} \in \mathbb{C}^{N \times n}$ be any matrix such that

$$
\operatorname{colspan} V_{n}=\mathcal{K}_{n}(M, R) .
$$

Then the transfer function (40), $H_{n}$, of the reduced-order model (37) defined by the projected data matrices (42) satisfies:

$$
H_{n}(s)=H(s)+\mathcal{O}\left(\left(s-s_{0}\right)^{j}\right) .
$$

If in addition, the expansion point $s_{0}$ is chosen to be real,

$$
s_{0} \in \mathbb{R},
$$

then the matrices (45), $M$ and $R$, are real and the basis matrix $V_{n}$ in (49) can be constructed to be real. In fact, any of the usual Krylov subspace algorithms for constructing basis vectors for $\mathcal{K}_{n}(M, R)$, such as the band Lanczos method or the band Arnoldi process [16], generate a real basis matrix $V_{n}$. In this case, as mentioned at the end of Section 5.1, the transfer function $H_{n}$ is positive real, and thus the PRIMA reduced-order models are passive.

On the other hand, the data matrices (42) of the PRIMA reduced-order models are full in general, and thus, PRIMA does not preserve the special block structure of the original data matrices (23).

\section{The SPRIM algorithm}

In this section, we describe the SPRIM algorithm [17, 20], which unlike PRIMA, preserves the block structure of the data matrices (23). 


\subsection{The projection theorem}

It turns out that in order to guarantee a Padé-type property (50) of the reduced-order transfer function, the condition (49) on the matrix $V_{n}$ can be relaxed. In fact, let $\hat{V} \in \mathbb{C}^{N \times \hat{n}}$ be any matrix with the property

$$
\mathcal{K}_{n}(M, R) \subseteq \operatorname{colspan} \hat{V} .
$$

Then the statement of Theorem 2 remains correct when (49) is replaced by the weaker condition (52). This result, which is sometimes referred to as the projection theorem, was derived by Grimme in [25]. A different proof of the projection theorem is given in [18, Theorem 8.6.1]. Note that, in view of (52), $\hat{V}$ must have at least as many columns as any matrix $V_{n}$ satisfying (49).

The projection theorem can be used to devise an order reduction algorithm that in the Padé-type sense (50), is at least as accurate as PRIMA, but unlike PRIMA preserves the block structure of the original data matrices (23). Indeed, let $V_{n}$ be any basis matrix of the $n$-th Krylov subspace $\mathcal{K}_{n}(M, R)$. Let

$$
V_{n}=\left[\begin{array}{l}
\hat{V}_{1} \\
\hat{V}_{2} \\
\hat{V}_{3}
\end{array}\right]
$$

be the partitioning of $V_{n}$ corresponding to the block sizes of the matrices $A$ and $E$ in (23), and formally set

$$
\hat{V}=\left[\begin{array}{ccc}
\hat{V}_{1} & 0 & 0 \\
0 & \hat{V}_{2} & 0 \\
0 & 0 & \hat{V}_{3}
\end{array}\right]
$$

Since $V_{n}$ is a basis matrix of $\mathcal{K}_{n}(M, R)$, the matrix (53) satisfies (52). Thus, we can replace $V_{n}$ by $\hat{V}$ in (42) and still obtain a reduced-order model (37) that satisfies a Padé-type property (50). In view of the block structures of the original data matrices (23) and of the matrix (53), the reduced-order matrices are of the form

$A_{n}=-\left[\begin{array}{ccc}\tilde{D}_{11} & \tilde{\mathcal{A}}_{l} & \tilde{\mathcal{A}}_{v} \\ -\tilde{\mathcal{A}}_{l}^{T} & 0 & 0 \\ -\tilde{\mathcal{A}}_{v}^{T} & 0 & 0\end{array}\right], \quad E_{n}=\left[\begin{array}{ccc}\tilde{M}_{11} & 0 & 0 \\ 0 & \tilde{L} & 0 \\ 0 & 0 & 0\end{array}\right], \quad B_{n}=\left[\begin{array}{cc}\tilde{\mathcal{A}}_{i} & 0 \\ 0 & 0 \\ 0 & -\hat{V}_{3}^{T}\end{array}\right]$,

and thus the block structure of the original data matrices (23) is now preserved. The resulting order reduction procedure is the most basic form of the SPRIM algorithm.

We remark that in this most basic form of SPRIM, the relative sizes of the blocks in (23) are not preserved. Recall that the sizes of the three diagonal blocks of $A$ and $E$ in (23) are the number of interconnections, the number of inductors, and the number of voltage sources, respectively, of the given RCL circuit. These numbers are very different in general. Typically, there are only 
very few voltage sources. Similarly, the number of inductors is typically significantly smaller than the number of interconnections. Consequently, unless $n$ is smaller than the number of voltage sources, the subblock $\hat{V}_{3}$ does not have full column rank. The sizes of the subblocks in the third block rows and columns of the reduced-order data matrices can thus be reduced further by replacing $\hat{V}_{3}$ with a matrix whose columns span the same subspace as $\hat{V}_{3}$, but which has full column rank, before the projection is performed. Similar size reductions are possible if $\hat{V}_{2}$ or $\hat{V}_{1}$ do not have full column rank.

\subsection{SPRIM}

The basic form of SPRIM, with possible size reduction of the subblocks $\hat{V}_{l}$, $l=1,2,3$, as an optional step, can be summarized as follows.

Algorithm 1 (SPRIM for general RCL circuits)

- Input: matrices of the form

$$
A=-\left[\begin{array}{ccc}
D_{11} & \mathcal{A}_{l} & \mathcal{A}_{v} \\
-\mathcal{A}_{l}^{T} & 0 & 0 \\
-\mathcal{A}_{v}^{T} & 0 & 0
\end{array}\right], \quad E=\left[\begin{array}{ccc}
M_{11} & 0 & 0 \\
0 & L & 0 \\
0 & 0 & 0
\end{array}\right], \quad B=\left[\begin{array}{cc}
\mathcal{A}_{i} & 0 \\
0 & 0 \\
0 & -I
\end{array}\right]
$$

where $D_{11}, M_{11} \succeq 0$;

an expansion point $s_{0} \in \mathbb{R}$.

- Formally set

$$
M=\left(s_{0} E-A\right)^{-1} E, \quad R=\left(s_{0} E-A\right)^{-1} B .
$$

- Until $n$ is large enough, run your favorite block Krylov subspace method (applied to $M$ and $R$ ) to construct the columns of the basis matrix

$$
V_{n}=\left[\begin{array}{llll}
v_{1} & v_{2} & \cdots & v_{n}
\end{array}\right]
$$

of the $n$-th block Krylov subspace $\mathcal{K}_{n}(M, R)$, i.e.,

$$
\operatorname{colspan} V_{n}=\mathcal{K}_{n}(M, R) .
$$

- Let

$$
V_{n}=\left[\begin{array}{l}
\hat{V}_{1} \\
\hat{V}_{2} \\
\hat{V}_{3}
\end{array}\right]
$$

be the partitioning of $V_{n}$ corresponding to the block sizes of $A$ and $E$.

- (Optional step) For $l=1,2,3$ do:

If $r_{l}:=\operatorname{rank} \hat{V}_{l}<n$, determine an $N \times r_{l}$ matrix $\tilde{V}_{l}$ with

$$
\operatorname{colspan} \hat{V}_{l}=\operatorname{colspan} \tilde{V}_{l}, \quad \operatorname{rank} \tilde{V}_{l}=r_{l},
$$

and set $\hat{V}_{l}:=\tilde{V}_{l}$. 
- Set

$$
\begin{aligned}
& \tilde{D}_{11}=\hat{V}_{1}^{*} D_{11} \hat{V}_{1}, \quad \tilde{\mathcal{A}}_{l}=\hat{V}_{1}^{*} \mathcal{A}_{l} \hat{V}_{2}, \quad \tilde{\mathcal{A}}_{v}=\hat{V}_{1}^{*} \mathcal{A}_{v} \hat{V}_{3}, \\
& \tilde{M}_{11}=\hat{V}_{1}^{*} M_{11} \hat{V}_{1}, \quad \tilde{L}=\hat{V}_{2}^{*} L \hat{V}_{2}, \quad \tilde{\mathcal{A}}_{i}=\hat{V}_{1}^{*} \mathcal{A}_{i} .
\end{aligned}
$$

- Output: the data matrices

$$
\begin{aligned}
A_{n}=- & {\left[\begin{array}{ccc}
\tilde{D}_{11} & \tilde{\mathcal{A}}_{l} & \tilde{\mathcal{A}}_{v} \\
-\tilde{\mathcal{A}}_{l}^{T} & 0 & 0 \\
-\tilde{\mathcal{A}}_{v}^{T} & 0 & 0
\end{array}\right], \quad E_{n}=\left[\begin{array}{ccc}
\tilde{M}_{11} & 0 & 0 \\
0 & \tilde{L} & 0 \\
0 & 0 & 0
\end{array}\right], } \\
B_{n} & =\left[\begin{array}{cc}
\tilde{\mathcal{A}}_{i} & 0 \\
0 & 0 \\
0 & -\hat{V}_{3}^{T}
\end{array}\right]
\end{aligned}
$$

of the SPRIM reduced-order model

$$
\begin{aligned}
E_{n} \frac{d}{d t} \tilde{x}(t) & =A_{n} \tilde{x}(t)+B_{n} u(t), \\
\tilde{y}(t) & =B_{n}^{T} \tilde{x}(t),
\end{aligned}
$$

We remark that the main computational cost of the SPRIM algorithm is running the block Krylov subspace method to obtain $\hat{V}_{n}$. This is the same as for PRIMA. Thus generating the PRIMA reduced-order model and the SPRIM reduced-order model $H_{n}$ involves the same computational costs. Implementation details of the SPRIM algorithm can be found in [20].

\section{Padé-type approximation property of SPRIM}

While PRIMA and SPRIM generate different reduced-order models, the projection theorem suggests that both models have comparable accuracy in the sense of the Padé-type approximation property (50). However, as long as the expansion point $s_{0}$ is chosen to be real, cf. (51), numerical experiments show that SPRIM is significantly more accurate than PRIMA; see the numerical results in Section 8. This higher accuracy is a consequence of the structure preservation of the SPRIM reduced-order data matrices (54). We stress that the restriction (51) of the expansion point $s_{0}$ to real values is needed anyway for both PRIMA and SPRIM, in order to guarantee that the PRIMA and SPRIM reduced-order models are passive.

For the special case of RCL circuits with current sources only, which means that the third block rows and columns in (54) are not present, it was proven in [18, Theorem 8.7.2] that the SPRIM reduced-order transfer function satisfies (50) with $j$ replaced by $2 j$.

A recent result [19] shows that the higher accuracy of SPRIM holds true in the more general context of Padé-type model order reduction of $J$-Hermitian 
linear dynamical systems. A square matrix $A$ is said to be $J$-Hermitian with respect to a given nonsingular matrix $J$ of the same size as $A$ if

$$
J A=A^{*} J .
$$

Clearly, for RCL circuits, in view of (23), the original data matrices $A$ and $E$ are $J$-Hermitian with respect to the indefinite matrix

$$
J=\left[\begin{array}{ccc}
I & 0 & 0 \\
0 & -I & 0 \\
0 & 0 & -I
\end{array}\right] .
$$

Furthermore, due to the structure preservation of SPRIM, the reduced-order data matrices $A_{n}$ and $E_{n}$ in (54) are $J_{n}$-Hermitian with respect to a matrix $J_{n}$ of the same form as $J$, but with correspondingly smaller blocks. Finally, the matrix (53), $\hat{V}$, which is used to generate the SPRIM models, satisfies the compatibility condition

$$
J \hat{V}=\hat{V} J_{n} .
$$

The result in [19] shows that for $J$-Hermitian data matrices and $J_{n}$-Hermitian reduced-order data matrices, the compatibility condition implies the higher accuracy of Padé-type reduced-order models. In particular, as a special case of this more general result, we have the following theorem.

Theorem 3. Let $n=n(j)$ be of the form (48) for some $1 \leq j \leq j_{\max }$, and assume that $s_{0} \in \mathbb{R}$. Then the transfer function (40), $H_{n}$, of the SPRIM reduced-order model (37) defined by the projected data matrices (54) satisfies:

$$
H_{n}(s)=H(s)+\mathcal{O}\left(\left(s-s_{0}\right)^{2 j}\right) .
$$

\section{Numerical examples}

In this section, we present results of some numerical experiments with the SPRIM algorithm. These results were first reported in [17]. The results in this section illustrate the higher accuracy of the SPRIM reduced-order models vs. the PRIMA reduced-order models.

\subsection{A PEEC circuit}

The first example is a circuit resulting from the so-called PEEC discretization [32] of an electromagnetic problem. The circuit is an RCL network consisting of 2100 capacitors, 172 inductors, 6990 inductive couplings, and a single resistive source that drives the circuit. The circuit is formulated as a 2-port. We compare the PRIMA and SPRIM models corresponding to the same dimension $n$ of the underlying block Krylov subspace. The expansion point $s_{0}=2 \pi \times 10^{9}$ was used. In Figure 1, we plot the absolute value of the $(2,1)$ 
component of the $2 \times 2$-matrix-valued transfer function over the frequency range of interest. The dimension $n=120$ was sufficient for SPRIM to match the exact transfer function. The corresponding PRIMA model of the same dimension, however, has not yet converged to the exact transfer function in large parts of the frequency range of interest. Figure 1 clearly illustrates the better approximation properties of SPRIM due to matching of twice as many moments as PRIMA.

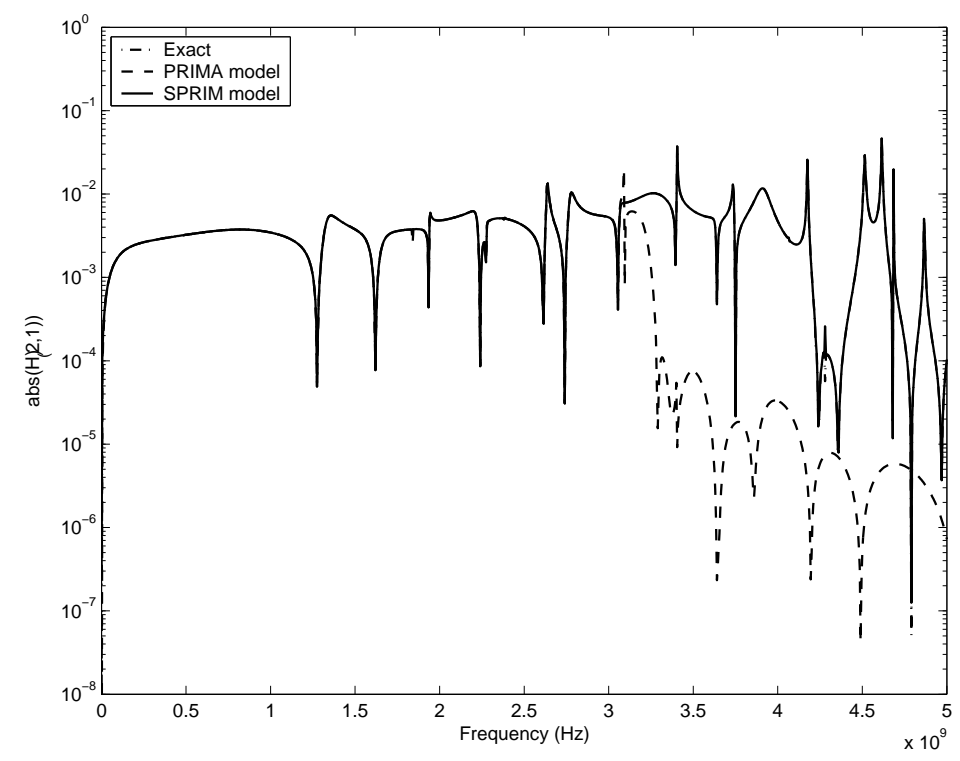

Fig. 1. $\left|H_{2,1}\right|$ for PEEC circuit

\subsection{A package model}

The second example is a 64-pin package model used for an RF integrated circuit. Only eight of the package pins carry signals, the rest being either unused or carrying supply voltages. The package is characterized as a 16-port component ( 8 exterior and 8 interior terminals). The package model is described by approximately 4000 circuit elements, resistors, capacitors, inductors, and inductive couplings. We again compare the PRIMA and SPRIM models corresponding to the same dimension $n$ of the underlying block Krylov subspace. The expansion point $s_{0}=5 \pi \times 10^{9}$ was used. In Figure 2, we plot the absolute value of one of the components of the $16 \times 16$-matrix-valued transfer function over the frequency range of interest. The state-space dimension $n=80$ was sufficient for SPRIM to match the exact transfer function. The corresponding 
PRIMA model of the same dimension, however, does not match the exact transfer function very well near the high frequencies; see Figure 3.

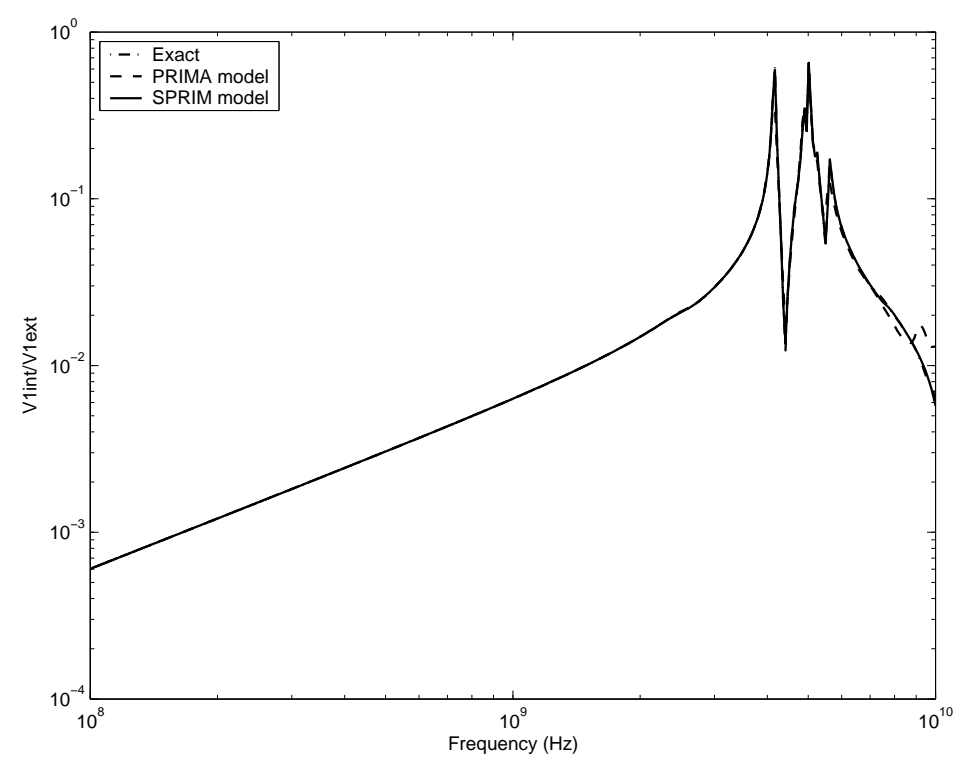

Fig. 2. The package model

\subsection{A mechanical system}

Exploiting the equivalence (see, e.g., [27]) between RCL circuits and mechanical systems, both PRIMA and SPRIM can also be applied to reduced-order modeling of mechanical systems. Such systems arise for example in the modeling and simulation of MEMS devices. In Figure 4, we show a comparison of PRIMA and SPRIM for a finite-element model of a shaft. The expansion point $s_{0}=\pi \times 10^{3}$ was used. The dimension $n=15$ was sufficient for SPRIM to match the exact transfer function in the frequency range of interest. The corresponding PRIMA model of the same dimension, however, has not converged to the exact transfer function in large parts of the frequency range of interest. Figure 4 again illustrates the better approximation properties of SPRIM due to the matching of twice as many moments as PRIMA.

\section{Concluding remarks}

In this paper, we reviewed the formulation of general RCL circuits as linear dynamical systems and discussed the problem of structure-preserving model 


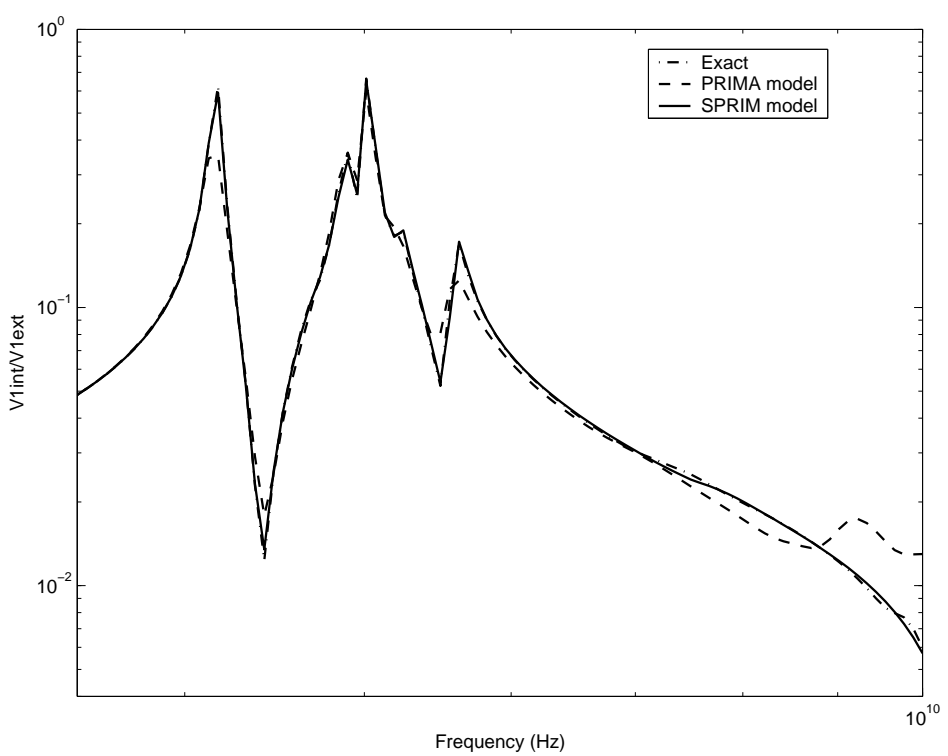

Fig. 3. The package model, high frequencies

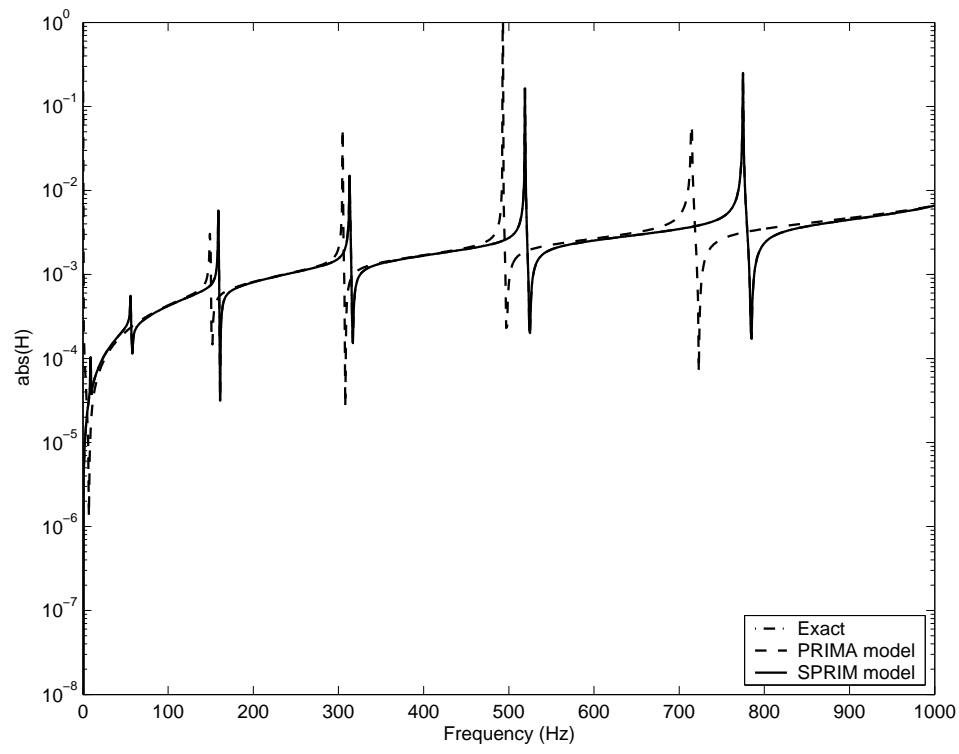

Fig. 4. A mechanical system 
reduction of such systems. We described the general framework of order reduction via projection and discussed two state-of-the-art projection algorithms, namely PRIMA and SPRIM.

While there has been a lot of progress in Krylov subspace-based structurepreserving model reduction of large-scale linear dynamical systems in recent years, there are still many open problems. All state-of-the-art structurepreserving methods, such as SPRIM, first generate a basis matrix of the underlying Krylov subspace and then employ explicit projection using some suitable partitioning of the basis matrix to obtain a structure-preserving reduced-order model. In particular, there are two major problems with the use of such explicit projections. First, it requires the storage of the basis matrix, which becomes prohibitive in the case of truly large-scale linear dynamical systems. Second, the approximation properties of the resulting structure-preserving reduced-order models are far from optimal, and they show that the available degrees of freedom are not fully used. It would be highly desirable to have structure-preserving reduction method that do no involve explicit projection and would thus be applicable in the truly large-scale case. Other unresolved issues include the automatic and adaptive choice of suitable expansion points $s_{0}$ and robust and reliable stopping criteria and error bounds.

\section{Acknowledgement}

This work was supported in part by the National Science Foundation through Grant DMS-0613032.

\section{References}

1. J. I. Aliaga, D. L. Boley, R. W. Freund, and V. Hernández. A Lanczos-type method for multiple starting vectors. Math. Comp., 69:1577-1601, 2000.

2. B. D. O. Anderson and S. Vongpanitlerd. Network Analysis and Synthesis. Prentice-Hall, Englewood Cliffs, New Jersey, 1973.

3. W. E. Arnoldi. The principle of minimized iterations in the solution of the matrix eigenvalue problem. Quart. Appl. Math., 9:17-29, 1951.

4. Z. Bai, P. Feldmann, and R. W. Freund. How to make theoretically passive reduced-order models passive in practice. In Proc. IEEE 1998 Custom Integrated Circuits Conference, pages 207-210, Piscataway, New Jersey, 1998. IEEE.

5. G. A. Baker, Jr. and P. Graves-Morris. Padé Approximants. Cambridge University Press, New York, New York, second edition, 1996.

6. M. Benzi, G. H. Golub, and J. Liesen. Numerical solution of saddle point problems. Acta Numerica, 14:1-137, 2005.

7. N. Deo. Graph Theory with Applications to Engineering and Computer Science. Prentice-Hall, Englewood Cliffs, New Jersey, 1974.

8. I. M. Elfadel and D. D. Ling. Zeros and passivity of Arnoldi-reduced-order models for interconnect networks. In Proc. 34nd ACM/IEEE Design Automation Conference, pages 28-33, New York, New York, 1997. ACM. 
9. P. Feldmann and R. W. Freund. Efficient linear circuit analysis by Padé approximation via the Lanczos process. In Proceedings of EURO-DAC'94 with EURO-VHDL '94, pages 170-175, Los Alamitos, California, 1994. IEEE Computer Society Press.

10. P. Feldmann and R. W. Freund. Efficient linear circuit analysis by Padé approximation via the Lanczos process. IEEE Trans. Computer-Aided Design, 14:639-649, 1995.

11. P. Feldmann and R. W. Freund. Reduced-order modeling of large linear subcircuits via a block Lanczos algorithm. In Proc. 32nd ACM/IEEE Design Automation Conference, pages 474-479, New York, New York, 1995. ACM.

12. P. Feldmann and R. W. Freund. Interconnect-delay computation and signalintegrity verification using the SyMPVL algorithm. In Proc. 1997 European Conference on Circuit Theory and Design, pages 132-138, Los Alamitos, California, 1997. IEEE Computer Society Press.

13. R. W. Freund. Passive reduced-order models for interconnect simulation and their computation via Krylov-subspace algorithms. In Proc. 36th ACM/IEEE Design Automation Conference, pages 195-200, New York, New York, 1999. ACM.

14. R. W. Freund. Reduced-order modeling techniques based on Krylov subspaces and their use in circuit simulation. In B. N. Datta, editor, Applied and Computational Control, Signals, and Circuits, volume 1, pages 435-498. Birkhäuser, Boston, 1999.

15. R. W. Freund. Krylov-subspace methods for reduced-order modeling in circuit simulation. J. Comput. Appl. Math., 123(1-2):395-421, 2000.

16. R. W. Freund. Model reduction methods based on Krylov subspaces. Acta Numerica, 12:267-319, 2003.

17. R. W. Freund. SPRIM: structure-preserving reduced-order interconnect macromodeling. In Tech. Dig. 2004 IEEE/ACM International Conference on Computer-Aided Design, pages 80-87, Los Alamitos, California, 2004. IEEE Computer Society Press.

18. R. W. Freund. Padé-type model reduction of second-order and higher-order linear dynamical systems. In P. Benner, V. Mehrmann, and D. C. Sorensen, editors, Dimension Reduction of Large-Scale Systems, Lecture Notes in Computational Science and Engineering, Vol. 45, pages 191-223, Berlin/Heidelberg, 2005. Springer-Verlag.

19. R. W. Freund. On Padé-type model order reduction of $J$-Hermitian linear dynamical systems. Technical report, Department of Mathematics, University of California, Davis, California, 2007. Available online at

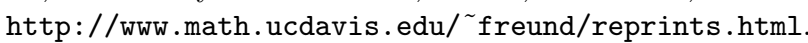

20. R. W. Freund. The SPRIM algorithm for structure-preserving order reduction of general RCL circuits. Technical report, Department of Mathematics, University of California, Davis, California, 2008. In preparation.

21. R. W. Freund and P. Feldmann. Reduced-order modeling of large passive linear circuits by means of the SyPVL algorithm. In Tech. Dig. 1996 IEEE/ACM International Conference on Computer-Aided Design, pages 280-287, Los Alamitos, California, 1996. IEEE Computer Society Press.

22. R. W. Freund and P. Feldmann. Small-signal circuit analysis and sensitivity computations with the PVL algorithm. IEEE Trans. Circuits and Systems-II: Analog and Digital Signal Processing, 43:577-585, 1996. 
23. R. W. Freund and P. Feldmann. The SyMPVL algorithm and its applications to interconnect simulation. In Proc. $199^{7}$ International Conference on Simulation of Semiconductor Processes and Devices, pages 113-116, Piscataway, New Jersey, 1997. IEEE.

24. R. W. Freund and P. Feldmann. Reduced-order modeling of large linear passive multi-terminal circuits using matrix-Padé approximation. In Proc. Design, Automation and Test in Europe Conference 1998, pages 530-537, Los Alamitos, California, 1998. IEEE Computer Society Press.

25. E. J. Grimme. Krylov projection methods for model reduction. PhD thesis, Department of Electrical Engineering, University of Illinois at Urbana-Champaign, Urbana-Champaign, Illinois, 1997.

26. C. Lanczos. An iteration method for the solution of the eigenvalue problem of linear differential and integral operators. J. Res. Nat. Bur. Standards, 45:255$282,1950$.

27. R. Lozano, B. Brogliato, O. Egeland, and B. Maschke. Dissipative Systems Analysis and Control. Springer-Verlag, London, 2000.

28. A. Odabasioglu. Provably passive RLC circuit reduction. M.S. thesis, Department of Electrical and Computer Engineering, Carnegie Mellon University, 1996.

29. A. Odabasioglu, M. Celik, and L. T. Pileggi. PRIMA: passive reduced-order interconnect macromodeling algorithm. In Tech. Dig. 1997 IEEE/ACM International Conference on Computer-Aided Design, pages 58-65, Los Alamitos, California, 1997. IEEE Computer Society Press.

30. A. Odabasioglu, M. Celik, and L. T. Pileggi. PRIMA: passive reduced-order interconnect macromodeling algorithm. IEEE Trans. Computer-Aided Design, 17(8):645-654, 1998.

31. L. T. Pillage and R. A. Rohrer. Asymptotic waveform evaluation for timing analysis. IEEE Trans. Computer-Aided Design, 9:352-366, 1990.

32. A. E. Ruehli. Equivalent circuit models for three-dimensional multiconductor systems. IEEE Trans. Microwave Theory Tech., 22:216-221, 1974.

33. L. M. Silveira, M. Kamon, I. Elfadel, and J. White. A coordinate-transformed Arnoldi algorithm for generating guaranteed stable reduced-order models of RLC circuits. In Tech. Dig. 1996 IEEE/ACM International Conference on Computer-Aided Design, pages 288-294, Los Alamitos, California, 1996. IEEE Computer Society Press.

34. J. Vlach and K. Singhal. Computer Methods for Circuit Analysis and Design. Van Nostrand Reinhold, New York, New York, second edition, 1994. 